\title{
In Vivo Proton Magnetic Resonance Spectroscopy for Metabolic Changes of Human Brain Edema
}

\author{
Kyousuke KAMADA, Kiyohiro HOUKIN, Yoshinobu IWASAKI, Hiroshi ABE, \\ and Takeshi KASHIWABA*
}

Department of Neurosurgery, Hokkaido University School of Medicine, Sapporo;
${ }^{*}$ Kashiwaba Neurosurgical Hospital, Sapporo

\begin{abstract}
The metabolism of the brain was investigated in eight patients with peritumoral edema, six patients with ischemic stroke, and 28 normal controls using proton magnetic resonance (MR) spectroscopy. The MR studies were performed using a 1.5-T whole-body imaging and spectroscopy system with a 1500-msec repetition time (TR) and a $270-\mathrm{msec}$ echo time (TE). The peak areas for $N$-acetyl-aspartate (NAA), choline-containing compounds (Cho), creatine and phosphocreatine (Cr), and lactate (Lac) were measured, and the $\mathrm{NAA} / \mathrm{Cr}, \mathrm{Cho} / \mathrm{Cr}$, and $\mathrm{Lac} / \mathrm{Cr}$ ratios were calculated. To quantify and compare the serial spectra, relaxation effects were investigated by acquisitions at two different points (TRs or TEs) and by monoexponential fitting. The normal $\mathrm{NAA} / \mathrm{Cr}$ and $\mathrm{Cho} / \mathrm{Cr}$ ratios were 2.76 and 1.09 , respectively. Lac could not be identified in normal brains. In ischemic stroke and peritumoral edema, significantly increased $\mathrm{Lac} / \mathrm{Cr}$ and decreased $\mathrm{NAA} / \mathrm{Cr}$ ratios were observed. Resolution of peritumoral edema was associated with normalized NAA/Cr ratio and disappearance of Lac. The $T_{1}$ relaxation times of the metabolites were similar in normal brain and peritumoral edema, but the $T_{2}$ values were significantly shortened. Serial measurements of $T_{2}$ values in two patients with peritumoral edema showed gradual normalization corresponding to improvement of the edema. To absolutely quantify metabolite concentrations in edema, changes in relaxation times should be considered.
\end{abstract}

Key words: brain edema, lactate, $N$-acetyl-aspartate, proton magnetic resonance spectroscopy

\section{Introduction}

Brain edema is defined as an abnormal accumulation of fluid within the brain parenchyma and can be classified into two different types depending on pathophysiological mechanism.9" The "cytotoxic" type is due to a severe disturbance of cell metabolism associated with anoxia and ischemia. Intracellular concentrations of molecules and sodium are increased, resulting in increased cellular volume and narrowing of the extracellular space. The "vasogenic" type is associated with brain tumors and brain injury resulting from a breakdown of the bloodbrain barrier to macromolecules without brain tissue breakdown. Fluid leaks out of brain vessels and accumulates in the extracellular space. Edema is most frequently associated with tumors and ischemic stroke lesions. Peritumoral edema is mainly

Received November 1, 1993; Accepted March 22, 1994 vasogenic edema, while almost all stroke lesions cause a combination of vasogenic and cytotoxic edema and result in brain tissue breakdown. ${ }^{2}$

Proton magnetic resonance ( $\left.{ }^{1} \mathrm{H}-\mathrm{MR}\right)$ spectroscopy allows investigation of specific metabolites within tissue in vivo, and has yielded important information about the pathophysiological manifestations of some brain diseases. ${ }^{1,4,5,11,16)}{ }^{1} \mathrm{H}-\mathrm{MR}$ spectroscopy of ischemic stroke shows characteristic prolonged increase of Jactate (Lac) levels and loss of $\mathrm{N}$-acetylaspartate (NAA), indicating "neuronal loss." "1,5,8) However, the metabolism of peritumoral vasogenic edema has not been evaluated. As differentiation between certain types of brain edema by computed tomography and MR imaging remains difficult, we hope to obtain additional information from ${ }^{1} \mathrm{H}-\mathrm{MR}$ spectroscopy.

We report our ${ }^{1} \mathrm{H}-\mathrm{MR}$ spectroscopy study of metabolic changes in brain edema, particularly peritumoral edema, and discuss the pathophysiological mechanisms causing the spectral changes 
corresponding to resolution of the edema.

\section{Materials and Methods}

Fourteen patients with brain edema, six with ischemic stroke and eight with peritumoral edema, and 28 normal controls (ranging from 22 to 68 years old) were examined. All ischemic stroke patients had clear, reliable histories with unequivocal clinical data typical of ischemic stroke, and neuroimaging evidence of acute lesions (Table 1). Serial MR imaging and MR angiography showed the main vessels were occluded in all the cases. All tumor patients had undergone tumor removal. Tissue specimen examination revealed meningioma in four cases, glioblastoma in two, metastatic brain tumor in one, and malignant lymphoma in one (Table 2). The peritumoral lesions demonstrated typical histological evidence of brain edema without tumor invasion.

MR imaging and spectroscopy used a 1.5-T Magnetom SP (Siemens, Erlangen, Germany), a whole-body scanner that combines imaging and spectroscopy systems. Multislice images in transverse (repetition time [TR] $2200 \mathrm{msec}$, echo time [TE] 80

Table 1 Clinical data and metabolite ratios in stroke edema patients

\begin{tabular}{ccccccccc}
\hline $\begin{array}{c}\text { Case } \\
\text { No. }\end{array}$ & $\begin{array}{c}\text { Age/ } \\
\text { Sex }\end{array}$ & $\begin{array}{c}\text { Poststroke } \\
\text { period } \\
\text { (hrs) }\end{array}$ & $\begin{array}{c}\text { Area of } \\
\text { infarction }\end{array}$ & & & & & \\
\cline { 1 - 4 } \cline { 6 - 8 } 1 & $78 / \mathrm{M}$ & 6 & It MCA & & 2.01 & 3.00 & 5.02 \\
2 & $61 / \mathrm{M}$ & 12 & rt MCA & & 1.99 & 2.00 & 4.50 \\
3 & $70 / \mathrm{F}$ & 6 & rt ICA & & 1.30 & 0.87 & 3.53 \\
4 & $85 / \mathrm{M}$ & 24 & rt ICA & & 1.10 & 1.18 & 2.11 \\
5 & $80 / \mathrm{F}$ & 12 & rt ICA & & 1.00 & 0.08 & 6.40 \\
6 & $74 / \mathrm{F}$ & 5 & It ICA & & 1.48 & 1.57 & 5.30 \\
\hline
\end{tabular}

ICA: internal carotid artery, MCA: middle cerebral artery.

Table 2 Clinical data and metabolite ratios in peritumoral edema patients

\begin{tabular}{rrllllll}
$\begin{array}{c}\text { Case } \\
\text { No. }\end{array}$ & $\begin{array}{c}\text { Age/ } \\
\text { Sex }\end{array}$ & $\begin{array}{c}\text { Histology } \\
\text { of tumor }\end{array}$ & $\begin{array}{c}\text { Location } \\
\text { of edema }\end{array}$ & NAA/Cr & Cho/Cr & Lac/Cr \\
\cline { 3 - 6 } 7 & $59 / \mathrm{F}$ & meningioma & rt P & 1.70 & 0.62 & 0.76 \\
8 & $62 / \mathrm{M}$ & metastasis & rt T & 2.88 & 1.93 & 1.91 \\
9 & $42 / \mathrm{F}$ & meningioma & rt P & 2.38 & 0.63 & 0.88 \\
10 & $61 / \mathrm{F}$ & lymphoma & rt T & 1.80 & 1.11 & 0.60 \\
11 & $27 / \mathrm{F}$ & meningioma & It P & 3.40 & 0.80 & 0 \\
12 & $66 / \mathrm{M}$ & glioblastoma & It F & 1.33 & 1.00 & 2.00 \\
13 & $42 / \mathrm{M}$ glioblastoma & It F & 1.91 & 1.05 & 0.11 \\
14 & $60 / \mathrm{F}$ & meningioma & rt T & 0.60 & 0.61 & 0 \\
\hline
\end{tabular}

F: frontal lobe, $\mathrm{P}$ : parietal lobe, $\mathrm{T}$ : temporal lobe. msec, 5-mm slice thickness) and coronal planes (TR $600 \mathrm{msec}$, TE $15 \mathrm{msec}, 5-\mathrm{mm}$ slice thickness) were then obtained to locate the volume of interest (VOI) for spectroscopy. Water-suppressed, localized ${ }^{1} \mathrm{H}$ spectra were obtained with a sequence of pointresolved spectroscopy. Control data were obtained from a volume in the left parietal region $(3 \times 3 \times 3$ $\mathrm{cm})$. The VOI was selected to contain the bulk of the peritumoral or stroke edema and avoid overlapping the tumor and surrounding bone marrow. Care was taken in serial examinations to select the same volume based on anatomical landmarks in the image, although the size and shape of the edema varied. The magnetic field over the VOI was homogenized by adjusting the linear shims to minimize the water signal width. A water signal width of less than $7 \mathrm{~Hz}$ was obtained from all the edematous lesions. The water signal was suppressed with chemical shift-selective pulses optimized for the detection of brain metabolites. The signals (free induction decays) of 100 acquisitions were obtained using standard parameters (TR $1500 \mathrm{msec}$, TE $270 \mathrm{msec}$ ). The data obtained were averaged, zero-filled to 2048 data points, and processed by fast Fourier transformation. The areas of the four main peaks due to NAA, choline-containing compounds (Cho), creatine and phosphocreatine $(\mathrm{Cr})$, and Lac were calculated using a computer algorithm with the lower and upper integration limits set manually. The NAA/Cr, Cho/ $\mathrm{Cr}$, and $\mathrm{Lac} / \mathrm{Cr}$ ratios were calculated. The $\mathrm{Cr}$ signal was chosen as an internal reference because the intensity appears to be relatively stable under different metabolic conditions in the brain. ${ }^{3,12,15)}$

Changes in the metabolite signal ratios depend on changes in concentration and in the relaxation rate of individual metabolites. Therefore, a total of four spectra were obtained using combinations of two TRs (1500 and $3000 \mathrm{msec}$ ) and two TEs (135 and 270 $\mathrm{msec})$. The relaxation times for the metabolites were estimated from the data using simple monoexponential fitting.

Data are presented as mean values $\pm \mathrm{SD}$. Student's $t$-test was used to assess significance, with $\mathrm{p}<0.001$ considered statistically significant.

\section{Results}

\section{Normal brain}

The peaks corresponding to NAA at $2.03 \mathrm{ppm}, \mathrm{Cr}$ at $3.02 \mathrm{ppm}$, and Cho at $3.20 \mathrm{ppm}$ were readily assigned as described previously, ${ }^{3,15}$ and were fairly constant in amplitude throughout the selected VOI. Lac could not be detected in normal brains. The $\mathrm{NAA} / \mathrm{Cr}$ and $\mathrm{Cho} / \mathrm{Cr}$ ratios were $2.76 \pm 0.48$ and 
Table 3 Comparison of metabolite ratios in edema and normal brain

\begin{tabular}{|c|c|c|c|}
\hline & $\mathrm{NAA} / \mathrm{Cr}$ & $\mathrm{Lac} / \mathrm{Cr}$ & $\mathrm{Cho} / \mathrm{Cr}$ \\
\hline $\begin{array}{l}\text { Normal brain } \\
(\mathrm{n}=28)\end{array}$ & $2.76 \pm 0.48$ & not detected & $1.09 \pm 0.13$ \\
\hline $\begin{array}{l}\text { Peritumoral edema } \\
\quad(\mathrm{n}=8)\end{array}$ & $1.92 \pm 0.94^{*}$ & $0.78 \pm 0.86^{*}$ & $0.97 \pm 0.44$ \\
\hline $\begin{array}{l}\text { Stroke edema } \\
\quad(\mathrm{n}=6)\end{array}$ & $1.48 \pm 0.44^{*}$ & $4.47 \pm 1.49^{*}$ & $1.57 \pm 0.83$ \\
\hline
\end{tabular}

$1.09 \pm 0.13$, respectively.

\section{Stroke edema}

The results of ${ }^{1} \mathrm{H}-\mathrm{MR}$ spectroscopy of ischemic stroke edema are given in Table 1. The $\mathrm{NAA} / \mathrm{Cr}$ ratio was significantly lower, and the $\mathrm{Lac} / \mathrm{Cr}$ ratio significantly higher compared to normal brain (Table 3). Figure 1 shows an MR image of the VOI and spectra for Case 6. Several months after onset, the spectrum shows only a slight Lac peak, and complete absence of NAA, Cr, and Cho.

\section{Peritumoral edema}

The results of 'H-MR spectroscopy of peritumoral edema are given in Table 2 . The NAA/Cr ratio was significantly lower, and the $\mathrm{Lac} / \mathrm{Cr}$ ratio significantly higher than in normal brain. However, the $\mathrm{Lac} / \mathrm{Cr}$ ratio was significantly lower than in ischemic stroke edema (Table 3).

Four (Cases 7-10) of the eight tumor patients could be followed up with serial ${ }^{1} \mathrm{H}-\mathrm{MR}$ spectroscopy. The Lac/Cr ratio rapidly decreased and the NAA/Cr ratio slowly increased to within normal limits (Fig. 2). Two patients also demonstrated considerable increases in the $\mathrm{Cho} / \mathrm{Cr}$ ratio (data not shown). Figure 3 shows typical MR images of the VOI and ${ }^{1} \mathrm{H}-\mathrm{MR}$ spectra before and after tumor removal in Case 10.

Normal $\mathrm{T}$, relaxation times for $\mathrm{Cho}, \mathrm{Cr}$, and NAA were 1250,1467 , and $1527 \mathrm{msec}$, respectively, while normal $T_{2}$ values were 343,267 , and $357 \mathrm{msec}$, respectively (Table 4$)$. In peritumoral edema the $T_{1}$ values were relatively close to normal values, but the $T_{2}$ values, particularly for NAA and Cho, were significantly shorter. Serial postoperative measurements of $T_{2}$ values in two patients with peritumoral edema showed gradual normalization of the $T_{2}$ values corresponding to resolution of the edema (data not shown).

\section{Discussion}

${ }^{1}$ H-MR spectroscopy gives a better signal-to-noise ratio and spatial resolution than phosphorus $\left({ }^{31} \mathrm{P}\right)$ MR spectroscopy, and is therefore a practical method for clinical use. 'H-MR spectroscopy can pro-
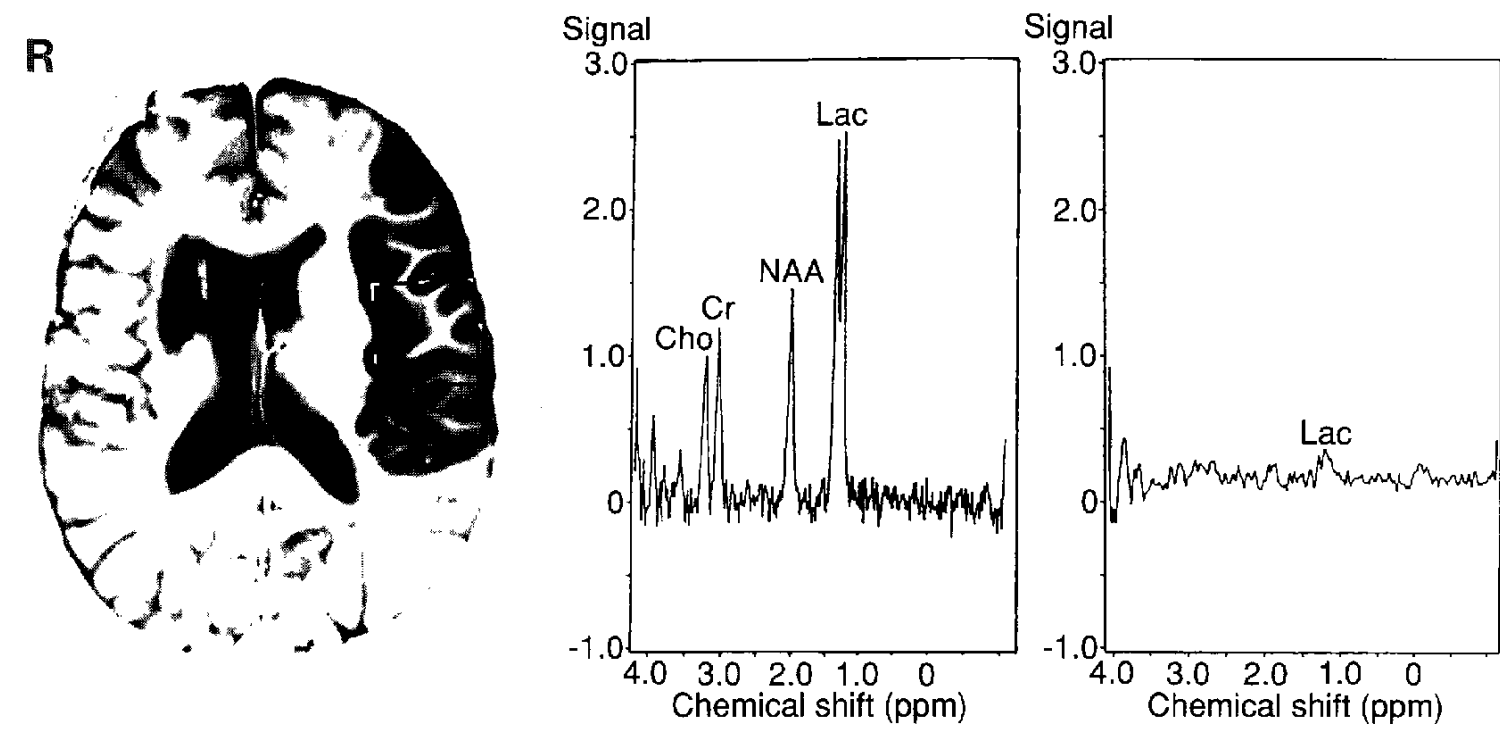

Fig. 1 Case 6 with acute ischemic stroke. left: $\mathrm{T}_{2}$-weighted transverse MR image, showing the VOI for ${ }^{1} \mathrm{H}$-MR spectroscopy in the lesion. center, right: ${ }^{1} \mathrm{H}-\mathrm{MR}$ spectra 6 hours after onset showing markedly increased Lac and decreased NAA signals (center), and 3 months later showing only the Lac signal (right). 

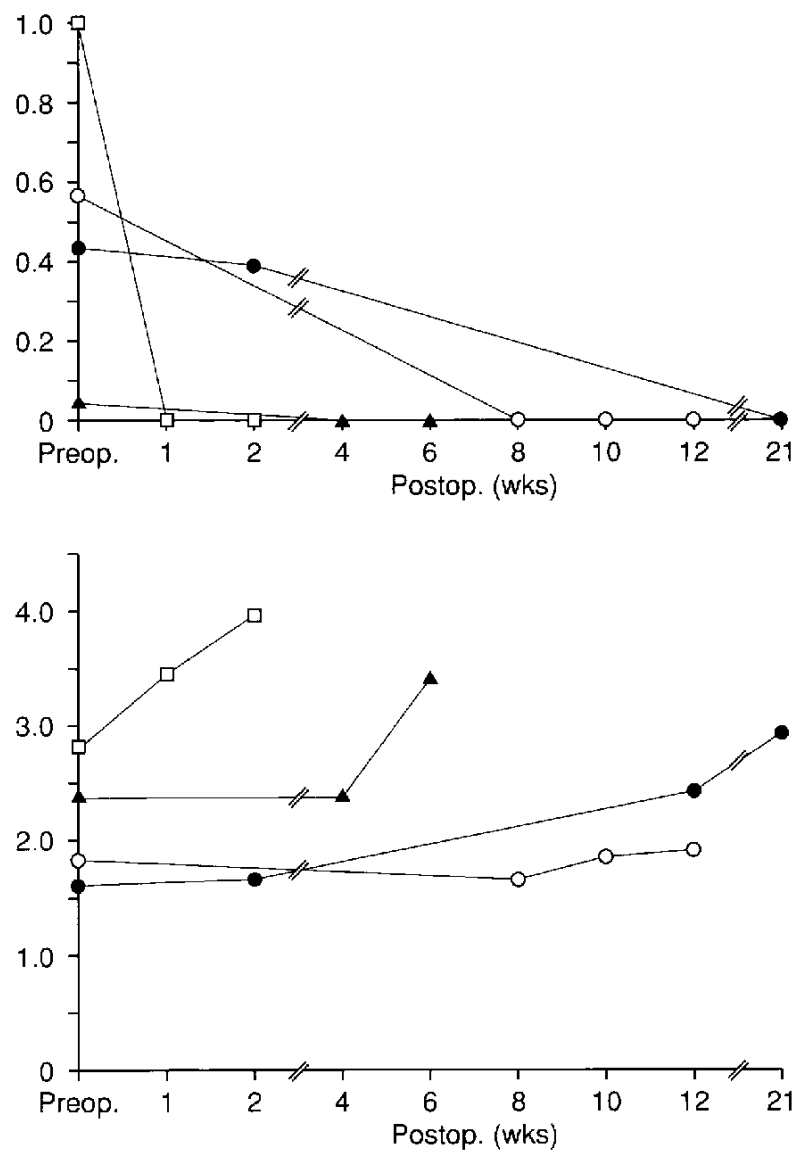

Fig. 2 Serial changes in Lac/Cr (upper) and NAA/Cr ratios (lower) in the edematous lesions of Cases $7(\bullet), 8(\square), 9(\Delta)$, and $10(0)$.

vide quantitative data about $\mathrm{Cr}$, Cho, Lac, and NAA in the human brain.

Creatine kinase, which is mainly present in neurons and muscles, converts creatine to creatine phosphate ( $\mathrm{PCr}$ ) although this process is easily reversed under certain conditions. ${ }^{31} \mathrm{P}-\mathrm{MR}$ spectroscopy only detects the concentration of $\mathrm{PCr}$ which easily changes. The physiological meaning of the $\mathrm{Cr}$ (creatine and $\mathrm{PCr}$ ) peak in ${ }^{1} \mathrm{H}-\mathrm{MR}$ spectroscopy is still unclear, but may be used as an internal reference because the intensity of the $\mathrm{Cr}$ peak is relatively stable in various diseases. ${ }^{3-5,14)}$

The Cho signal from the phosphoryl- and glycerophosphoryl-choline is an indicator of membrane synthesis and degeneration. ${ }^{4,12)}$ An increased Cho signal may reflect the active membrane metabolism associated with brain tumors ${ }^{4)}$ and demyelination in multiple sclerosis. ${ }^{11,16)}$ Whether the Cho signal is increased in cerebral infarction remains controversial. $^{5,12)}$ In our study, peritumoral and ischemic stroke edemas were associated with both increased and decreased Cho signals. The Cho signal may not be an indicator for peritumoral or stroke edema.

Lac is the end product of anaerobic metabolism and serves as a marker of glycolysis. Lac concentration is markedly increased in cerebral infarction, many brain tumors, and other diseases..$^{4,8,11,14,16)} \mathrm{A}$ previous study showed stroke patients had persistent high Lac levels for several months after onset. ${ }^{5}$ Our 'H-MR spectroscopy study of ischemic stroke edema generally confirms this tendency. Hossman ${ }^{7}$ and Hanstock et al. ${ }^{6)}$ demonstrated accumulation of Lac in experimental vasogenic edema using biochemical analysis and ${ }^{1} \mathrm{H}-\mathrm{MR}$ spectroscopy, respectively. Our study demonstrated that Lac accumulates in peritumoral edema. Our follow-up results suggest that Lac production stops after tumor removal and Lac is washed out and disappears from the lesions.

NAA, discovered by Tallan in $1956,{ }^{17}$ ) is found in higher concentrations in gray matter than in white matter, and rapidly disappears when neurotoxin kainic acid is injected into neurons. ${ }^{10)}$ NAA is considered to be a neuronal marker. Decreases in the $\mathrm{NAA} / \mathrm{Cr}$ ratio are considered to indicate neuronal loss in cerebral infarction, multiple sclerosis, and brain tumors. ${ }^{1,3-5,8,11,12,14,16)}$ Our study showed a decreased NAA/Cr ratio in acute edema, which normalized with resolution after treatment. It is quite difficult to explain what the increased NAA signal means since neuronal regeneration in the brain is not generally accepted. Nuclear MR signals are closely related to relaxation effects and the quantity of metabolites. The relaxation times we obtained in normal volunteers were almost the same as in previous reports. ${ }^{3,13)}$ Our study found the $T_{2}$ relaxation times of metabolites in peritumoral edema, particularly those of NAA, became shorter without significant changes in the $T_{1}$ values. The $T_{2}$ values may reflect "slow" overall motions of whole and bound molecules. Frahm et al. ${ }^{3)}$ claimed that longer $\mathrm{T}_{2}$ values of metabolites may reflect high mobility. A clear pathophysiological mechanism for the shortening of the $T_{2}$ value is difficult to propose, but the shorter $T_{2}$ value may reflect lower mobility of neuronal NAA in edematous brain. The decreased NAA/Cr ratio therefore indicates neuronal loss and lower NAA mobility. In contrast, the increase in the NAA/Cr ratio may only indicate greater mobility of the NAA.

${ }^{1} \mathrm{H}-\mathrm{MR}$ spectroscopy can demonstrate metabolic differences between peritumoral edema and ischemic stroke edema. Edematous lesion around tumors may 

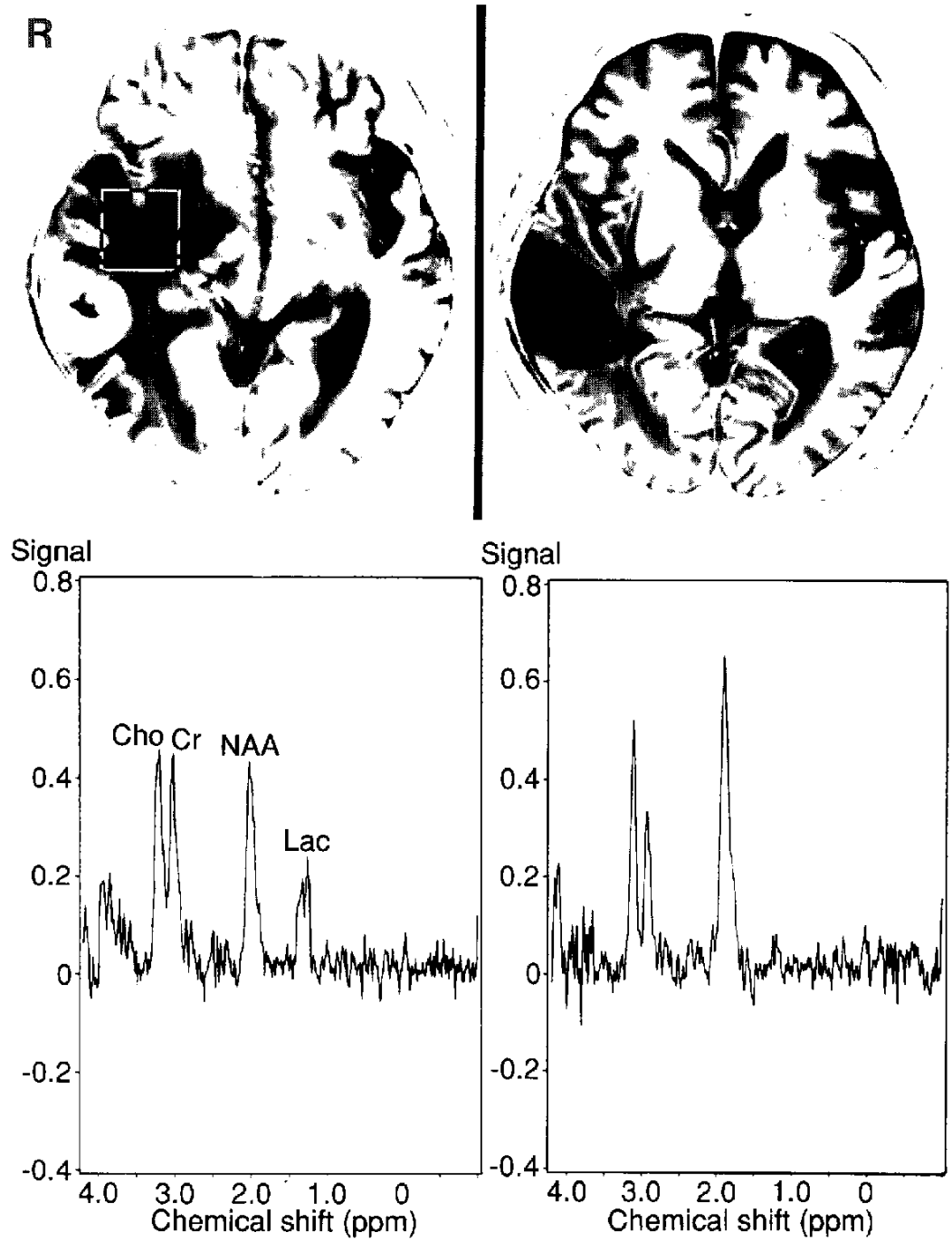

Fig. 3 Case 10 with brain edema associated with a malignant lymphoma. upper: $\mathrm{T}_{2}$-weighted transverse MR images before (left) and 2 weeks after surgery (right), showing the VOI for ${ }^{1} \mathrm{H}-\mathrm{MR}$ spectroscopy. lower: ${ }^{1} \mathrm{H}$-MR spectra before surgery showing lower NAA peak and slightly increased Lac peak (left), and 2 weeks after surgery showing increased NAA and disappearance of Lac signal (right).

Table $4 \quad T_{1}$ and $T_{2}$ relaxation times of major metabolites in normal brain and peritumoral edema

\begin{tabular}{|c|c|c|c|c|c|c|}
\hline & \multicolumn{3}{|c|}{$\mathrm{T}_{1}$ relaxation time (msec) } & \multicolumn{3}{|c|}{$\mathrm{T}_{2}$ relaxation time (msec) } \\
\hline & Cho & $\mathrm{Cr}$ & NAA & Cho & $\mathrm{Cr}$ & NAA \\
\hline Nor & $1250 \pm 250$ & $1467 \pm$ & $1527 \pm 145$ & $343 \pm 25$ & $267 \pm 69$ & $357 \pm 28$ \\
\hline Peritumoral edema & $1450 \pm 135$ & $1524 \pm 515$ & $1477 \pm 559$ & $138 \pm 45^{*}$ & $170 \pm 60$ & $210 \pm 52^{*}$ \\
\hline
\end{tabular}

Values are mean \pm SD. ${ }^{*}$ Significantly different $(\mathrm{p}<0.001)$ from normal brain.

have a ${ }^{1} \mathrm{H}-\mathrm{MR}$ spectrum with intermediate metabolite ratios between normal and infarcted brain and may recover completely after successful treatment. However, absolute quantification of metabolites in edematous lesions requires consideration of changes in relaxation time. 


\section{References}

1) Bruhn H, Frahm J, Gyngell ML, Merboldt $\mathrm{KD}$, Hänicke W, Sauter R: Cerebral metabolism in man after acute stroke: New observations using localized proton NMR spectroscopy. Magn Reson Med 9: 126131,1989

2) Fishman RA: Brain edema. $N$ Engl J Med 293: 706711,1975

3) Frahm J, Bruhn H, Gyngell ML, Merboldt KD, Hänicke W, Sauter R: Localized proton NMR spectroscopy in different regions of the human brain in vivo. Relaxation times and concentrations of cerebral metabolites. Magn Reson Med 11: 47-63, 1989

4) Gill SS, Thomas DGT, Bruggen NV, Gadian DG, Peden CJ, Bell JD, Cox IJ, Menon DK, Iles RA, Bryant DJ, Coutts GA: Proton MR spectroscopy of intracranial tumours: In vivo and vitro studies. $J$ Comput Assist Tomogr 14: 497-504, 1990

5) Graham GD, Blamire AM, Howseman AM, Rothman DL, Fayad PB, Brass LM, Petroff OAC, Shulman RG, Prichard JW: Proton magnetic resonance spectroscopy of cerebral lactate and other metabolites in stroke patients. Stroke 23: 333-340, 1992

6) Hanstock CC, Boisvert DPJ, Bendall MR, Allen PS: In vivo assessment of focal brain lactate alterations with NMR proton spectroscopy. J Cereb Blood Flow Metab 8: 208-214, 1988

7) Hossman KA: The pathophysiology of experimental brain edema. Neurosurg Rev 12: 263-280, 1989

8) Houkin K, Kwee IL, Nakada T: Persistent high lactate level as a sensitive MR spectroscopy indicator of completed infarction. $J$ Neurosurg 72: 763-766, 1990

9) Klatzo I: Neuropathological aspects of brain edema. $J$ Neuropath Exp Neurol 26: 1-14, 1967
10) Koller KJ, Zaczek R, Coyle J: N-acetyl-aspartylglutamate: Regional levels in rat brain and the effects of brain lesions as determined by a new HPLC method. $J$ Neurochem 43: 1136-1142, 1984

11) Matthews PM, Francis G, Antel J, Arnold DL: Proton magnetic resonance spectroscopy for metabolic characterization of plaques in multiple sclerosis. Neurology 41: 1251-1256, 1991

12) Miller BL: A review of chemical issues in ${ }^{1} \mathrm{H}$ NMR spectroscopy: $\mathrm{N}$-acetyl-aspartate, creatine and choline. NMR Biomed 4: 47-52, 1991

13) Narayana PA, Johnston D, Flamig DP: In vivo proton magnetic resonance spectroscopy studies of human brain. Magn Reson Imaging 9: 303-308, 1991

14) Peeling J, Wong D, Sutherland GR: Nuclear magnetic resonance study of regional metabolism after forebrain ischemia in rats. Stroke 20: 633-640, 1989

15) Petroff OAC, Ogino T, Alger JR: High-resolution proton magnetic resonance spectroscopy of rabbit brain: Regional metabolite levels and postmortem changes. J Neurochem 51: 163-171, 1988

16) Richards TL: Proton MR spectroscopy in multiple sclerosis: Value in establishing diagnosis, monitoring progression, and evaluating therapy. AJR 157: 10731078, 1991

17) Tallan HH: Studies on the distribution of N-acetylL-asparatic acid in brain. $J$ Biol Chem 224: 41-45, 1956

Address reprint requests to: K. Kamada, M.D., Department of Neurosurgery, Hokkaido University School of Medicine, North-15, West-7, Kita-ku, Sapporo 060, Japan. 